Behavioral/Cognitive

\title{
Ordinality and the Nature of Symbolic Numbers
}

\author{
Ian M. Lyons ${ }^{1,2}$ and Sian L. Beilock ${ }^{1}$ \\ ${ }^{1}$ University of Chicago, Chicago, Illinois 60637, and 2 Department of Psychology, University of Western Ontario, London, Ontario N6A 3K7, Canada
}

The view that representations of symbolic and nonsymbolic numbers are closely tied to one another is widespread. However, the link between symbolic and nonsymbolic numbers is almost always inferred from cardinal processing tasks. In the current work, we show that considering ordinality instead points to striking differences between symbolic and nonsymbolic numbers. Human behavioral and neural data show that ordinal processing of symbolic numbers (Are three Indo-Arabic numerals in numerical order?) is distinct from symbolic cardinal processing (Which of two numerals represents the greater quantity?) and nonsymbolic number processing (ordinal and cardinal judgments of dot-arrays). Behaviorally, distance-effects were reversed when assessing ordinality in symbolic numbers, but canonical distance-effects were observed for cardinal judgments of symbolic numbers and all nonsymbolic judgments. At the neural level, symbolic number-ordering was the only numerical task that did not show number-specific activity (greater than control) in the intraparietal sulcus. Only activity in left premotor cortex was specifically associated with symbolic number-ordering. For nonsymbolic numbers, activation in cognitive-control areas during ordinal processing and a high degree of overlap between ordinal and cardinal processing networks indicate that nonsymbolic ordinality is assessed via iterative cardinality judgments. This contrasts with a striking lack of neural overlap between ordinal and cardinal judgments anywhere in the brain for symbolic numbers, suggesting that symbolic number processing varies substantially with computational context. Ordinal processing sheds light on key differences between symbolic and nonsymbolic number processing both behaviorally and in the brain. Ordinality may prove important for understanding the power of representing numbers symbolically.

\section{Introduction}

Many have suggested that symbolic numbers (e.g., Indo-Arabic numerals) are derived from and are closely tied to an approximate number system (ANS) important for representing nonsymbolic numbers (e.g., dot-arrays) (Verguts and Fias, 2004; Nieder and Dehaene, 2009). Evidence for this view usually falls along three lines of reasoning. First, behavioral response curves when making judgments about symbolic and nonsymbolic numbers tend to show qualitatively similar patterns. For example, in both symbolic and nonsymbolic numbers, when the numerical difference between two numbers is small, it is more difficult to distinguish those numbers than when this difference is large (Buckley and Gillman, 1974; Dehaene, 2008; referred to as the distance-effect). Second, neuroimaging evidence often points to similar neural substrates for symbolic and nonsymbolic number processing (Fias et al., 2003; Diester and Nieder, 2007, 2010; Piazza et al., 2007; Eger et al., 2009). Third, one's ability to resolve quantities in the ANS is related to more complex symbolic math abilities (Halberda et al., 2008; Gilmore et al., 2010; Piazza et al., 2010; Lyons and Beilock, 2011; Wagner and Johnson, 2011; McCrink and Spelke, 2010).

However, an important caveat to the work supporting a strong link between symbolic and nonsymbolic number repre-

\footnotetext{
Received April 28, 2013; revised Sept. 4, 2013; accepted Sept. 10, 2013.

Author contributions: I.M.L. and S.L.B. designed research; I.M.L. performed research; I.M.L. analyzed data; I.M.L. and S.L.B. wrote the paper.

This work was funded by NSF CAREER DRL-0746970 and NSF Spatial Intelligence Learning Center to Sian Beilock.

Correspondence should be addressed to lan M. Lyons, Department of Psychology, University of Western Ontario, 361 Windermere Road, Office 307E, London, 0N N6G 3K7, Canada. E-mail: ilyons@uwo.ca.

DOI:10.1523/JNEUROSCI.1775-13.2013

Copyright $\odot 2013$ the authors $\quad 0270-6474 / 13 / 3317052-10 \$ 15.00 / 0$
}

sentations is that it has focused almost exclusively on cardinality. Cardinality answers the question, How many? All of the studies mentioned above employed paradigms-passive or activewhere subjects' focus was on relative changes in cardinality. Another key property of numbers is ordinality. Ordinality answers the question, What position? The ordinality of a given number tells you which number came previously, and which number comes next. In essence, ordinality tells you how a number relates to its closest neighbors.

We propose that a focus on cardinality has largely driven the conclusion that symbolic and nonsymbolic number representation is strongly linked. We contend that if one focuses instead on ordinality, important differences between symbolic and nonsymbolic number representation emerge. Ordinal information is positional in nature, and thus may be driven more by associations among elements rather than the magnitudes of the elements themselves (Turconi et al., 2006; Franklin and Jonides, 2009; Lyons and Beilock, 2009; Nieder, 2009). These associations may be differentially available, depending on the nature of numerical representations. Specifically, ordinal associations between numbers may be stronger and more readily available to symbolic than nonsymbolic numbers (see also Nieder, 2009), perhaps due to frequent recitation of the count-list.

In the current work, we use both behavioral and neural measures to test the hypothesis that the wide-spread conclusion regarding a tight link between symbolic and nonsymbolic number representations does not hold when ordinality is the focus. Further, given behavioral indications that ordinal and cardinal processing within symbolic numbers operates in qualitatively different ways (Turconi et al., 2006; Franklin and Jonides, 2009), we 


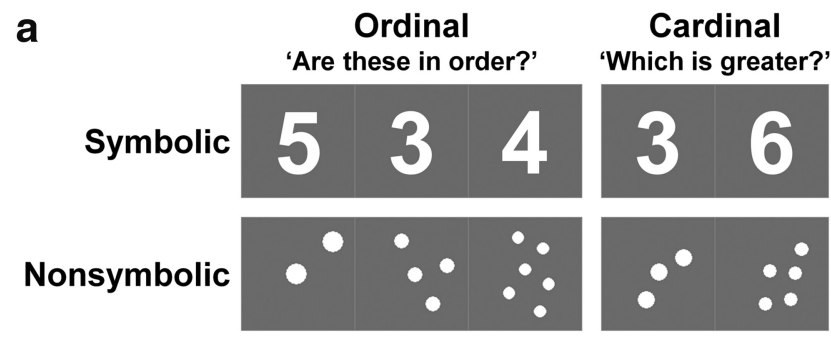

b

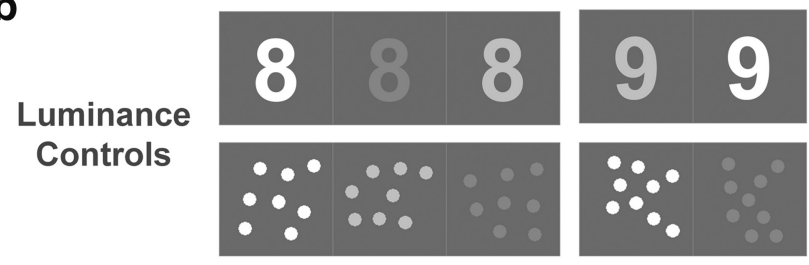

Figure 1. Examples of each experimental and control task. $\boldsymbol{a}$, Numerical tasks. The top row shows symbolic (numeral) conditions: Symbolic Ordering (NumOrd) and Symbolic Comparison (NumCard). The bottom row shows nonsymbolic (dot) conditions: Nonsymbolic Ordering (DotOrd) and Nonsymbolic Comparison (DotCard). $\boldsymbol{b}$, Luminance (control) tasks. To identify neural areas specific to each type of numerical processing in $\boldsymbol{a}$, activity during each experimental task in $\boldsymbol{a}$ was contrasted with activity during the corresponding control task in $\boldsymbol{b}$ : Luminance Ordering (LumOrd ${ }_{\text {num }}$ ), Luminance Comparison (LumCard ${ }_{\text {num }}$ ), Luminance Ordering (LumOrd dot , Luminance Comparison (LumCard ${ }_{\text {dot }}$ ).

tested (1) the neural underpinnings of this distinction, and (2) whether this distinction holds for nonsymbolic numbers as well.

\section{Materials and Methods}

Participants. Participants were 33 right-handed (16 female), neurologically normal University of Chicago students (age: $18.1-22.2$ years, $\sigma=$ 1.3 years).

Procedure. Participants completed three sessions. The first session was a behavioral prescreening session. Participants completed a working memory task [Reading-Span (Unsworth et al., 2005)], a reduced version of the ordering and comparison tasks (see below) they would perform in the scanner, and several other general cognitive assessments. Task-order was counterbalanced across participants; further, participants did not know the purpose of the study, and they did not know which, if any, of the tasks would be repeated in the scanner. Dot-array stimuli used in the prescreening session were not repeated while in the scanner. Participants also completed a survey battery that included the Edinburgh handedness inventory (Oldfield, 1971), basic demographic information, fMRI safety information, and several additional questionnaires. Participants who were deemed unsafe or unfit for scanning $(n=4)$, left-handed $(n=5)$, performed at chance on any of the tasks $(n=4)$, or had abnormally low working-memory (one participant was $>3$ SDs below the mean) were paid for their participation to that point but not allowed to continue to the scanning phase.

Approximately 1 week after the prescreening session, participants completed the main fMRI session. Upon arrival, participants completed a refresher program in which they practiced the ordering and comparison tasks. Dot-array stimuli were not repeated in the refresher, which in turn were unique with respect to the fMRI session stimuli.

Participants also completed several runs of a delayed match-to-sample task for another experiment, which is not analyzed here. Approximately 1 week after the first scanning session, participants returned for a second scanning session in which they performed a mental-arithmetic and a difficulty-matched verbal task. Data from this second scanning session are not analyzed here, as they were part of another study. After completing this last session, participants were thanked, paid, and fully debriefed as to the purpose(s) of the study.

Scanning procedure. Participants completed five functional runs of ordering and comparison tasks: four experimental (number) and four control (luminance) tasks (Fig. 1). There were two blocks of four trials of
Table 1. Behavioral means for each condition

\begin{tabular}{|c|c|c|c|c|}
\hline & \multicolumn{2}{|l|}{ Ordering } & \multicolumn{2}{|l|}{ Comparison } \\
\hline & Experimental & Control & Experimental & Control \\
\hline \multicolumn{5}{|c|}{ Error rates (\% incorrect) } \\
\hline Numerals & $9.0(1.0)$ & $5.6(0.9)$ & $4.3(0.9)$ & $1.5(0.4)$ \\
\hline Dots & $18.2(1.1)$ & $4.7(0.9)$ & $7.6(0.9)$ & $1.6(0.3)$ \\
\hline \multicolumn{5}{|c|}{ Response times (ms) } \\
\hline Numerals & $952(41)$ & $752(22)$ & $554(18)$ & $511(15)$ \\
\hline Dots & $1034(35)$ & $796(25)$ & $672(24)$ & $530(17)$ \\
\hline
\end{tabular}

Numbers in parentheses are SEs.

each task in a given run (time between blocks: 4-12 s). Before each block, a $1.5 \mathrm{~s}$ cue noted the nature of the upcoming task. Stimuli for each trial were presented for $500 \mathrm{~ms}$, after which the screen remained blank for $2 \mathrm{~s}$ or until response (intertrial-interval: $1 \mathrm{~s}$ ). No feedback was given during the scanning session.

Our conditions of interest may be divided along two orthogonal axes (Fig. 1a). One axis is cardinal versus ordinal. Cardinal judgments were made by assessing which of two numbers represents the greater quantity; ordinal judgments were made by assessing whether three numbers were in order. "In order" in the current study meant that all three items are either in increasing or decreasing (left-right) order. The second axis is symbolic versus nonsymbolic number representation. Here, symbolic means Indo-Arabic numerals; nonsymbolic means arrays of dots presented too quickly to count.

To assess both neural differences and similarities between conditions specific to number processing, we also included four control conditions (Fig. 1b). The control conditions involved the same judgments as the corresponding experimental condition (ordinal, cardinal), but in terms of relative luminance; the type of visual stimulus (numerals, dots) was also matched with experimental conditions. The control conditions were thus designed to remove three cognitive factors from the resulting activity patterns: visual input, response demands, and the decision process. Including these control tasks in each of those conjunctions ensures that the observed neural overlap is less likely to stem from the general cognitive processes noted above than if one merely assessed common activation above baseline.

In the ordinal tasks, participants judged whether three stimuli were in increasing/decreasing or mixed order. If all three stimuli were in (leftright) increasing or decreasing order, subjects pressed a button with one index finger. If the three stimuli were in some other "mixed" order, they pressed a button with their other index finger. For each ordinal task, a fourth of trials was increasing, a fourth was decreasing, and the remaining half was in mixed order. Which finger indicated which response was counterbalanced across participants; the response-mapping was held constant for a given participant across all sessions. All stimuli were white on a neutral gray background. Quantities were 1-9 with distances of $1[\max (n)-\operatorname{medi}$ $\operatorname{an}(n)=\operatorname{median}(n)-\min (n)=1]$ or 2 . For the luminance-ordering control-tasks, stimulus luminance varied between white and the neutralgray background. The quantities represented by the dot-arrays and numerals were constant for each of the three luminance stimuli in a given trial, which was selected randomly from the integers 1-9. Luminance permutations followed the same pattern as in the number-ordering tasks above.

In the cardinal tasks, participants judged which of two stimuli was numerically greater (quantity-comparison) or brighter (luminancecomparison). If the stimulus on the left was the correct answer, participants were to press a button with their left index finger; if the stimulus on the right was correct, participants were to press a button with their index finger. Quantities were 1-9 with (absolute) distances 1 and 2. Stimulus specifics for the luminance-comparison control-tasks followed those of the luminance-ordering tasks described above, with the exception that only two items were shown on a given trial.

Mean response-times (ms) and error-rates (\%-wrong) are shown in Table 1. The two measures showed consistent results across tasks (i.e., effects were always in the same direction), so the behavioral analyses that follow use a composite measure (for each subject and task, responsetimes and error-rates were each standardized-across all tasks, to pre- 
serve relative between-task differences-and then the standardized scores were averaged together). Note that using a composite measure (1) halves the number of tests required, thereby reducing the likelihood of type I errors, (2) puts all tasks on the same scale, which eliminates the temptation to conclude post hoc that one measure is important for this task and the other for that task, etc., and (3) implicitly controls for variation in speed/accuracy trade-offs across tasks.

All pairwise behavioral contrasts between an experimental task and its corresponding control task were significant at $p<0.001$ [effect-sizes were as follows: NumOrd-LumOrd ${ }_{\text {num }}: d=2.87$, DotOrd-LumOrd ${ }_{\text {dot }}$ : $d=4.70$, NumCard-LumCard ${ }_{\text {num }}: d=1.58$, DotCard-LumCard ${ }_{\text {dot }}:$ $d=3.94]$. Recall that the control tasks were designed to remove three cognitive factors from the resulting activity patterns: visual input, response demands, and the decision process (ordinal vs comparison decision); however, equating performance across tasks was not a central concern. This is because the field of psychology has operated for over a century on the assumption that differences in behavior (in this case, task-performance) are psychologically meaningful. Moving to equate the tasks behaviorally may thus inadvertently skew differences observed at the neural level. It was for this reason that we chose to design our control tasks to eliminate certain known confounds first, and address the issue of performance differences as a secondary concern. The central question, then, is whether the observed behavioral differences are driven by aspects of the processes with which one is concerned, or due to some extraneous "domain general" factors.

To address this issue, we tested whether differences in performance between a numerical task and its corresponding luminance-control could be explained by individual variation in a well-established measure of working memory capacity [Reading-Span (Unsworth et al., 2005)]. Working memory is a quintessentially domain-general cognitive capacity that captures one's ability to hold information in mind while mentally manipulating some other information. If, after accounting for working memory capacity, the difference in performance between two tasks remains significant, one can be more confident that this behavioral difference is indicative of representations or processes endemic to the cognitive phenomena ostensibly under investigation (in our case, numerical processing).

We first assessed the sign of the correlation between working memory and the difference between each numerical task and its corresponding luminance-control, which were as follows: NumOrd-LumOrd num $_{\text {: }}$ : $r_{(31)}=0.314, p=0.075$; DotOrd-LumOrd $\mathrm{dot}: r_{(31)}=-0.297, p=0.093$; NumCard-LumCard ${ }_{\text {num }}: r_{(31)}=0.205, p=0.252$; DotCard-LumCard dot $_{\text {: }}$ : $r_{(31)}=-0.122, p=0.499$. If a positive correlation is observed, individuals with higher working memory are more likely to show a larger difference between conditions, and those with lower working memory tend to show smaller differences. In that case, in the second step, we test whether the difference between tasks remains if we adjust everyone's difference scores to be as if each person is relatively low in working memory. Choosing a relatively low working memory score drives a more conservative approach because it works against our ability to detect differences between a given numerical task and its corresponding luminance-control condition. We chose to center on the 25th percentile of working memory scores, because this struck a balance between the need for a reliable estimate (an estimate closer to the middle of the distribution will be more reliable than an estimate from the edge of the distributione.g., the lowest score) and the need for a relatively low working memory score (the 25th percentile is clearly in the lower half of the distribution). Note that if a negative relation is observed between working memory and the difference between a numerical task and its control condition, this flips the relation-higher working-memory individuals tend to show smaller differences between conditions. In that case, to maintain a more conservative approach, we instead centered working memory on the 75 th percentile. Note that while subtracting different constants from the working memory covariate does not change its correlation with the difference in performance, it does change its influence on said difference (Delaney and Maxwell, 1981). As we are concerned with the latter point in this analysis, for this reason, in addition to the aim of adopting a more conservative approach, selection of one's zero-point is nonarbitrary. Note also that true mean-centering (i.e., at approximately the 50th percentile) is mathematically problematic when using a within-subjects ANCOVA [see the study by Delaney and Maxwell (1981) for details].

After controlling for working memory in the manner described above, all effects remained highly significant at $p<0.001$, with the

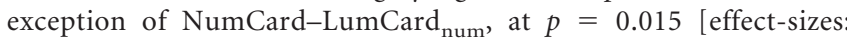
NumOrd-LumOrd num $: d=1.83$, DotOrd-LumOrd ${ }_{\text {dot }}: d=3.38$, NumCard-LumCard num $: d=0.93$, DotCard-LumCard dot $: d=2.89]$. Although accounting for working memory reduced effect sizes somewhat, the large differences in performance remained significant in all cases, which argues against a predominantly domain-general explanation for differences driven by "just" difficulty.

Imaging parameters and considerations. fMRI data were collected at the University of Chicago Hospital using a 3-tesla Philips-Achieva scanner and an 8-channel Philips SENSE head-coil. A standard echo-planar imaging sequence was used with a TR of $2000 \mathrm{~ms}(\mathrm{TE}=25 \mathrm{~ms})$. Thirty-six descending interleaved slices were acquired per temporal volume, with slice thickness of $3.0 \mathrm{~mm}$ ( $0.25 \mathrm{~mm}$ skip), in-plane matrix of $80 \times 80$ pixels with resolution of $2.875 \times 2.875 \mathrm{~mm}(\mathrm{FOV}=230 \times 230 \times 116.75$ $\mathrm{mm}$ ), and flip-angle of $80^{\circ}$. Before analysis, time-series were corrected for slice-timing, then subject motion, and then subjected to a high-pass temporal-filter (GLM Fourier basis set). After coregistration to anatomical images, images were spatially smoothed using a $3 \mathrm{~mm}$ full-width at half-maximum Gaussian kernel.

Data were next submitted to a random-effects GLM (Friston et al., 1994) with eight main predictors of interest (convolved using a standard 2-gamma HRF model) corresponding to the task-blocks described above. In each voxel and for each subject, parameter estimates for each subject and each task were submitted for second level analysis as described below. Preprocessing and RFX contrast analyses were conducted using BrainVoyager QX (version 2.4.1). Whole-brain results were thresholded first voxelwise at $p<0.005$, and subsequently cluster-level corrected for multiple-comparisons using a Monte-Carlo simulation procedure (Forman et al., 1995) at $\alpha<0.01$. Where region-of interest (ROI) statistics are reported, mean activity in a given ROI for a given task was calculated by first averaging the activity estimates across voxels in a given subject. Group-wise means and standard-errors were then calculated across subjects.

\section{Results}

\section{(Reverse) distance-effects}

The distance-effect is one of the most robust effects in the study of number processing, especially for nonsymbolic numbers (Moyer and Landauer, 1967). Previous behavioral work has shown that, while the distance effect is present for symbolic numbers when assessing cardinality (Buckley and Gillman, 1974; Lyons and Beilock, 2009), it can in some cases be reversed when assessing ordinality (Turconi et al., 2006; Franklin and Jonides, 2009). One interpretation of the reverse distance-effect is that it taps into direct retrieval mechanisms in the symbolic count-list (subjects are faster to retrieve a sequential ordered pair $\{23\}$ than a nonsequential ordered pair $\left.\left\{\begin{array}{ll}1 & 3\end{array}\right\}\right)$. The nature of the distance-effect is not known when assessing ordinality in nonsymbolic numbers. In the present dataset, we not only explore whether there are reverse-distance effects in nonsymbolic numbers, but also directly compare these effects with those seen in the cardinal and ordinal assessment of symbolic numbers. In this way, we can behaviorally test the hypothesis that ordinal assessment in symbolic numbers relies on retrieval mechanisms that are not available to nonsymbolic numbers.

Table 2 reports distance-effects for the numerical tasks (Fig. 1a). Statistics below are computed for composite scores (Table 2, left), but one can see from the rest of the table that patterns were consistent across all measures. As noted above, count-lists may play an important role in driving reversed distance-effects. We thus examined distance effects for the ordinality task in two dif- 
Table 2. Behavioral distance effects for each of the numerical tasks, with trials on the ordering tasks divided into mixed (Ord-Mix) and properly (Ord-Ord) ordered conditions (Card indicates cardinal tasks)

\begin{tabular}{|c|c|c|c|c|c|c|c|c|c|}
\hline & \multicolumn{3}{|c|}{ Composite } & \multicolumn{3}{|c|}{ Response times (ms) } & \multicolumn{3}{|c|}{ Error-rates (\% incorrect) } \\
\hline & Card & Ord-Mix & Ord-Ord & Card & Ord-Mix & Ord-Ord & Card & Ord-Mix & Ord-Ord \\
\hline \multirow[t]{2}{*}{ Numerals } & 0.09 & 0.21 & -0.35 & 13 & 30 & -57 & 1.5 & 3.6 & -5.6 \\
\hline & $(0.05)$ & $(0.09)$ & $(0.13)$ & (6) & $(20)$ & $(20)$ & (1.1) & (1.7) & $(2.7)$ \\
\hline \multirow[t]{2}{*}{ Dots } & 0.57 & 0.38 & 0.39 & 69 & 49 & 35 & 10.0 & 6.7 & 7.5 \\
\hline & $(0.07)$ & $(0.10)$ & $(0.14)$ & (11) & (18) & (21) & (1.4) & $(2.5)$ & $(2.6)$ \\
\hline
\end{tabular}

Composite scores were computed as follows: for each subject and task, response-times and error-rates were each standardized — across all tasks, to preserve relative between-task differences — and then the standardized scores for the two measures were averaged together (see Materials and Methods). Note that in all cases, a positive value indicates a canonical distance-effect and a negative value indicates a reversed distance-effect. Figures in parentheses are SEs.

ferent contexts: when all three numbers were in increasing/decreasing order (Ord-Ord: e.g., $\left\{\begin{array}{lll}1 & 2 & 3\end{array}\right\}$ or $\left\{\begin{array}{lll}3 & 2 & 1\end{array}\right\}$-i.e., as part of a familiar count-list), and when the three numbers were in mixed order (Ord-Mix: e.g., $\left\{\begin{array}{lll}2 & 1 & 3\end{array}\right\}$ or $\left.\left\{\begin{array}{lll}3 & 1 & 2\end{array}\right\}\right)$. In keeping with convention, performance on "far" (distance $=2$ in the current study) were subtracted from "close" (distance $=1$ ) trials, such that a positive value indicates a canonical distance-effect and a negative value indicates a reversed distance-effect.

For cardinal processing ("Card" in Table 2), we observed canonical distance-effects regardless of format. Consistent with previous work, in the current dataset, there was a robust distanceeffect for nonsymbolic numbers $(p<0.001)$, a weak effect for symbolic numbers $(p=0.091)$, and a notable difference between the two $(p<0.001)$. The pattern of results for the ordinal tasks on trials with mixed order (Ord-Mix) was similar: distance-effects obtained for both nonsymbolic $(p<0.001)$ and symbolic numbers ( $p=0.017$; the difference between effects was not significant in this case: $p=0.226$ ).

By contrast, when numbers were properly ordered according to an increasing/decreasing count-list (Ord-Ord), distanceeffects for symbolic numbers were reversed $(p=0.011)$, such that performance was better on trials with distances of 1 (e.g., $\{567\}$ ) than on trials with distances of 2 (e.g., $\left.\left\{\begin{array}{lll}4 & 6 & 8\end{array}\right\}\right)$. Crucially, distance-effects for nonsymbolic numbers in this condition were not reversed $(p<0.001)$, such that performance was better on trials with distances of 2 (e.g., $\{468\}$ ) than on trials with distances of 1 (e.g., $\left\{\begin{array}{l}5 \\ 6\end{array} 7\right\}$ ). Numeral and dot distance-effects in the OrdOrd condition were significantly different from one another $(p<$ $0.001)$, as were distance-effects across the Ord-Mix and Ord-Ord conditions when just numerals were considered $(p=0.001)$. Finally, the 2(Representation: Numerals, Dots) $\times 2$ (Condition: Ord-Mix, Ord-Ord) interaction was also significant $\left(F_{(1,32)}=\right.$ 10.64, $p=0.003$ ).

To summarize, the effect of numerical distance is reversed when assessing properly ordered sets, but only for symbolic numbers. This is consistent with the hypothesis that ordinality operates in a fundamentally different way for symbolic than for nonsymbolic numbers. That reversed distance-effects in numerals were further specific to the Ord-Ord condition indicates that this difference arises in at least in part due to direct retrieval from ordinal associations among numbers in the count list. Conversely, the distance-effects for nonsymbolic numbers in the ordering conditions (Ord-Mix and Ord-Ord) did not vary ( $p=$ 0.962 ), and both were similar in magnitude to that seen for the DotCard task ( $p=0.142$ and $p=0.272$, respectively). One possibility, then, is that assessing ordinality on nonsymbolic numbers operates in a manner similar to assessing cardinality. Interestingly, these data also predict that ordinality and cardinality assessment may be qualitatively different for symbolic numbers [note also that distance-effects in the numeral Ord-Ord condition were significantly different from those seen in the
NumCard task $(p=0.007)]$. We next turn to the fMRI data to test these assertions at the neural level.

\section{Cardinal processing}

Many studies have shown that the intraparietal sulcus (IPS) is important for representation of both symbolic and nonsymbolic numbers (Fias et al., 2003; Diester and Nieder, 2007; Piazza et al., 2007; Eger et al., 2009); although as noted in the Introduction, the vast majority of these studies have focused on cardinality. We thus first sought to replicate these prior results (showing that the IPS is common to cardinal processing across symbolic and nonsymbolic numbers) at the whole-brain level using the conjunction of the two contrasts: NumCard $>$ Control (LumCard $\mathrm{L}_{\text {num }}$ ) and DotCard $>$ Control $\left(\right.$ LumCard $\left._{\mathrm{dot}}\right)$. This identified regions that were specific to numerical processing and common to symbolic and nonsymbolic cardinal processing (of numbers; right column of Fig. 1a).

This analysis revealed two regions: right anterior IPS (IPSa) and an early visual area [bilateral calcarine sulci (CLS)]. The IPSa Region is shown in Figure $2 a$; region details and means can be found in Table $3 a$. These results are thus consistent with prior results showing that cardinal processing of symbolic and nonsymbolic numbers overlap in the IPS. Prior researchers have interpreted this to indicate that symbolic and nonsymbolic number representation is derived from overlapping neural sources. However, within right IPSa, activity for the numeral-ordering task was not significantly different than the corresponding luminanceordering control task $(p=0.701)$, indicating that the role this region plays in the NumOrd task is not specific to numerical processing. In contrast, for nonsymbolic numbers, dot-ordering activity was greater than control $(p<0.001)$. This suggests the conclusion that symbolic and nonsymbolic numbers share a common source in the IPS may be at best incomplete-certainly with respect to ordinal processing. In the next section we thus assess which brain areas are central to processing ordinal information in symbolic (and nonsymbolic) numbers.

\section{Ordinal processing}

We first examined whether there were areas of the brain that showed common effects for symbolic and nonsymbolic ordinal processing via the conjunction: NumOrd $>$ Control $\left(\right.$ LumOrd $\left._{\text {num }}\right)$ and DotOrd $>$ Control $\left(\right.$ LumOrd $\left._{\mathrm{dot}}\right)$. This identified regions that were both specific to numerical processing and common to symbolic and nonsymbolic ordinal processing (of numbers; left column of Fig. 1a). Regions are shown outlined in orange in Figure $2 b$; region details and means can be found in Table $3 b$. The only significant effect was in a dorsoposterior section of the anterior cingulate cortex (ACCdp), a region not canonically associated with number processing. The lack of overlap in canonical number processing areas (e.g., IPS and lateral parietal areas such as the angular and supramarginal gyri) suggests that the neural process- 
ing of numerical order depends on whether the numbers are represented symbolically versus nonsymbolically.

To better understand the role of this ACCdp region in ordinal processing, we next examined the networks important for assessing ordinality in symbolic and nonsymbolic numbers separately. For symbolic ordinal processing (Fig. 1a, topleft), we identified three regions-all within premotor cortex-via the contrast NumOrd $>$ Control $\left(\right.$ LumOrd $\left._{\text {num }}\right)$ : rostral supplementary motor area (PreSMA), left dorsal premotor cortex (PMd), and left ventral premotor cortex (PMv). Regions are shown in yellow in Figure 3; anatomical region details can be found in Table $4 a$; condition means can be found in Table $5 a$, left. Note that no parietal regions were found even at the more liberal threshold, $p<0.05$ (cluster corrected at $\alpha<.05)$. A 2(Condition: number, control) $\times 2$ (Format: symbolic, nonsymbolic) interaction confirmed the specificity of $\mathrm{PMd} / \mathrm{PMv}$ for symbolic ordinal processing (PreSMA did not show an interaction): $\mathrm{Nu}-$ mOrd activity was greater than DotOrd activity, and DotOrd activity was not significantly greater than the corresponding control task (Table $5 a$, right). Consistent with the reverse distance-effects (Table 2), one interpretation of these results is thus that ordinality in symbolic numbers is processed via controlled retrieval of sequential visuomotor associations (Grafton et al., 1998; Wise and Murray, 2000; Hoshi and Tanji, 2007; O'Shea et al., 2007) (i.e., a count-list).

For nonsymbolic ordinal processing (Fig. $1 a$, bottom-left), we identified a predominantly right-lateralized network of regions via the contrast: DotOrd $>$ Control $\left(\right.$ LumOrd $\left._{\text {dot }}\right)$. Crucially, part of this network was the right IPSa, which completely subsumed the region identified above from the conjunction between symbolic and nonsymbolic cardinal processing (from Fig. 2a). Regions from this analysis are shown in red in Figure 3; anatomical region details can be found in Table $4 b$; condition means can be found in Table $5 b$, left. The 2(Condition: number, control) $\times 2$ (Format: symbolic, nonsymbolic) interaction confirmed the specificity for nonsymbolic ordinal processing in all regions [except left anterior inferior frontal gyrus (IFGa) ]: DotOrd activity was significantly greater than $\mathrm{Nu}$ mOrd activity in all but left IFGa, and NumOrd activity was not significantly greater than control in any region, with the exception of ACCd (Table $5 b$, right).

Note that the overlap between the PreSMA and ACCd regions provide further context to understand the ACCdp overlap for symbolic and nonsymbolic ordinal processing shown via the conjunction analysis in Figure $3 b$. Specifically, the common ACCdp region found in the conjunction analysis may reflect two spatially adjacent but functionally different processes, as if one were to

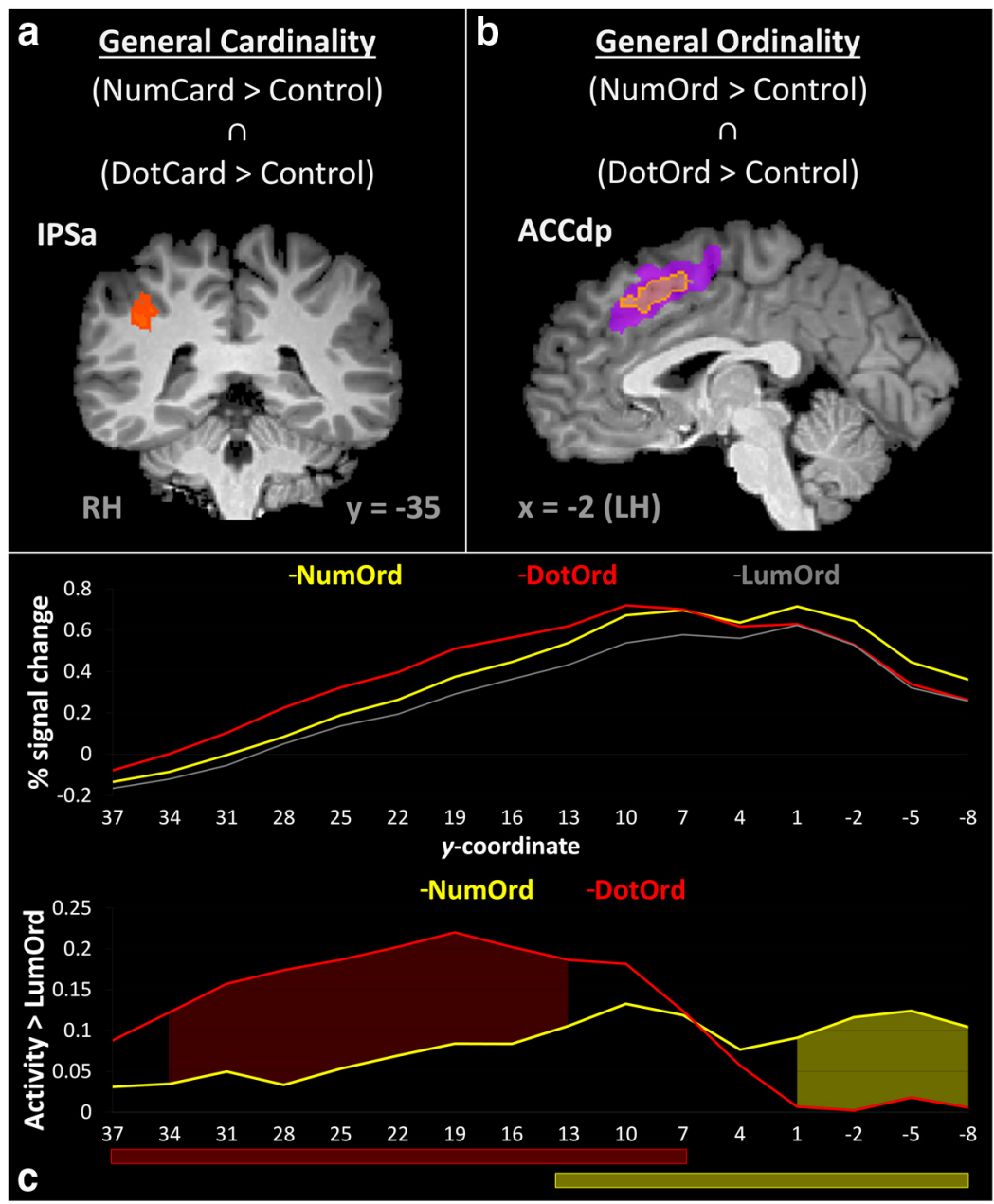

Figure 2. $\quad \boldsymbol{a}$ shows the right IPSa region. Cardinal processing activity was seen in this region for both symbolic (NumCard) and the orange outlined region for both symbolic and nonsymbolic numbers (conjunction of both tasks greater than control). The rger magenta region is the union of the two contrasts analyzed in c. c shows the anterior-posterior ( $y$-axis) shift in activity in the raph shows activity for Num0rd, DotOrd, and LumOrd (control) along an anterior-posterior gradient (y-coordinate). This is 列 shown together as a single line (Lum0rd, gray). The bottom graph shows the anterior-posterior crossover from Dot0rd to Num0rd more clearly. At each point, activity from the respective control task was subtracted from activity during each of the two experimental tasks, Num0rd and Dot0rd. The red and gold bars beneath the graph show where DotOrd and Num0rd tasks (respectively) showed significantly greater activity than control $(p<0.05)$. Shaded areas between the lines in the graph show where the difference between the two experimental tasks was significant $(p<0.05)$. a, Anterior; $p$, posterior; $d$, dorsal; $v$, ventral.

take a Venn diagram as a literal spatial metaphor. To quantify the anterior-posterior gradient implied by the contrasts shown in Figures 2-3, Figure $2 c$ shows ACC-PreSMA activity plotted as a function of the $y$-coordinate (anterior-posterior axis). To provide greater context, we first identified the larger region comprising the union of contrasts: NumOrd $>$ Control $\left(\operatorname{LumOrd}_{\text {num }}\right) \cup$ DotOrd $>$ Control $\left(\right.$ LumOrd $\left._{\text {dot }}\right)$, with each contrast thresholded at $p<0.05$ (corrected at $\alpha<.05$ ). This region is shown in magenta in Figure $3 b$ subsuming the stricter $(p<0.005, \alpha<.01)$ conjunction-region (orange). Within this larger, union ROI, for each subject and each condition, activity in all voxels in a given $y$-plane was averaged together. Note that this analysis constitutes a form of double-dipping (Kriegeskorte et al., 2009), in that a rough anterior-posterior difference between the DotOrd and NumOrd tasks is implied by the results in Figures 3-4. The following reconstrual of data (and in Fig. 2c) should thus be taken 
Table 3. Region details

\begin{tabular}{|c|c|c|c|c|c|c|c|c|c|}
\hline \multirow[b]{2}{*}{ Region } & \multicolumn{3}{|c|}{ Talairach coordinates } & \multirow[b]{2}{*}{ Vol. $\mathrm{mm}^{3}$} & \multirow[b]{2}{*}{ Condition } & \multicolumn{4}{|c|}{ \% Signal change: mean (SE) } \\
\hline & $x$ & $y$ & $z$ & & & Num0rd & DotOrd & NumCard & DotCard \\
\hline \multirow[t]{2}{*}{ R. IPSa ${ }^{a}$} & 37 & -35 & 35 & 391 & Number & $0.49(0.07)$ & $0.69(0.07)$ & $0.43(0.05)$ & $0.55(0.07)$ \\
\hline & & & & & Control & $0.51(0.06)$ & $0.49(0.07)$ & $0.33(0.04)$ & $0.34(0.06)$ \\
\hline \multirow[t]{2}{*}{$\operatorname{CLS}^{a}$} & 0 & -81 & 2 & 7593 & Number & $-0.03(0.06)$ & $0.01(0.05)$ & $0.05(0.05)$ & $0.15(0.05)$ \\
\hline & & & & & Control & $0.06(0.06)$ & $0.05(0.06)$ & $-0.13(0.07)$ & $-0.03(0.06)$ \\
\hline \multirow[t]{2}{*}{$\mathrm{ACCdp} p^{b}$} & -1 & 13 & 43 & 3289 & Number & $0.52(0.05)$ & $0.64(0.04)$ & $0.31(0.04)$ & $0.47(0.05)$ \\
\hline & & & & & Control & $0.41(0.05)$ & $0.40(0.05)$ & $0.29(0.06)$ & $0.29(0.05)$ \\
\hline
\end{tabular}

Anatomical information is shown on the left and mean neural activity on the right. Activity is shown for each experimental (Number) and control condition. a, Anterior; $p$, posterior; $d$, dorsal; $v$, ventral.

${ }^{a}$ Regions common to symbolic and nonsymbolic cardinality (see also Figure 2a).

${ }^{b}$ Regions common to symbolic and nonsymbolic ordinality (see also Figure $2 b$ ).

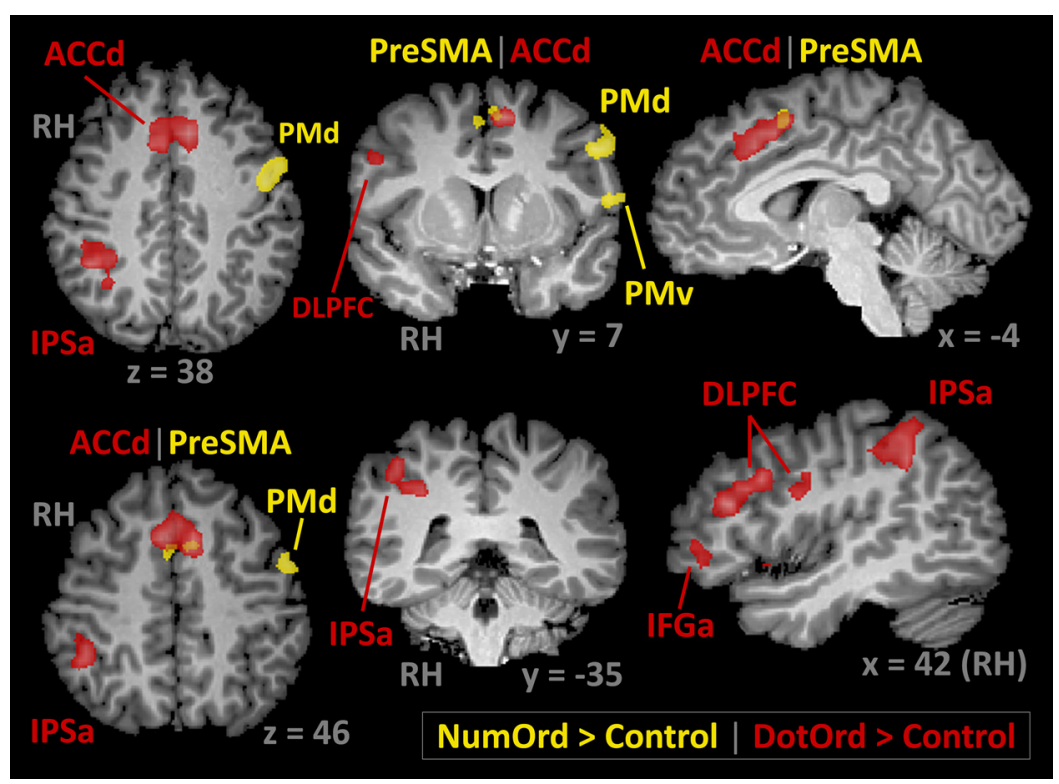

Figure 3. Regions with greater activity for symbolic number-ordering (Num0rd) relative to control in yellow, and regions with greater activity for nonsymbolic number-ordering (DotOrd) relative to control in red. DLPFC, Dorsal-lateral prefrontal cortex. $y=10$ and $y=1$. It was at approximately this point that the NumOrd and DotOrd conditions crossed one another. One can see this effect more clearly in the bottom graph of Figure $2 c$, where activity in the two experimental conditions is plotted relative to their respective control conditions. At anterior coordinates, DotOrd showed significantly greater activity than NumOrd until $\sim y=10$, at which point activity for DotOrd began to rapidly fall off and remained approximately equal to the control condition thereafter. By contrast, the NumOrd condition showed steadily greater activity than control (which became significant at approximately $y=13$ ) as the $y$-plane shifted posteriorly. The result was a crossover between DotOrd and $\mathrm{Nu}-$ mOrd activity, such that NumOrd showed significantly greater activity starting at $y=1$. In sum, anterior ACCd showed activity specific to ordinal processing of nonsymbolic numbers, and more posterior ACCd (and PreSMA) showed activity specific to ordinal

Table 4. Anatomical region details for regions specific to symbolic and nonsymbolic ordinal processing of numbers

\begin{tabular}{|c|c|c|c|c|}
\hline \multirow[b]{2}{*}{ Region } & \multicolumn{3}{|c|}{ Talairach coordinates } & \multirow[b]{2}{*}{ Vol. $\mathrm{mm}^{3}$} \\
\hline & $x$ & $y$ & $z$ & \\
\hline PresMA $^{a}$ & -1 & 9 & 48 & 362 \\
\hline L. $\mathrm{PMd}^{a}$ & -47 & 3 & 39 & 1647 \\
\hline L. PMv ${ }^{a}$ & -54 & 5 & 14 & 347 \\
\hline R. IPSa ${ }^{b}$ & 39 & -39 & 41 & 3172 \\
\hline $\mathrm{ACCd}^{b}$ & 1 & 19 & 41 & 5943 \\
\hline R. DLPFC ${ }^{b}$ & 44 & 19 & 27 & 4145 \\
\hline R. IFGa ${ }^{b}$ & 40 & 40 & 1 & 687 \\
\hline L. IFGa ${ }^{b}$ & -43 & 38 & 2 & 336 \\
\hline R. INSa ${ }^{b}$ & 31 & 19 & 3 & 1355 \\
\hline L. INSa ${ }^{b}$ & -32 & 19 & 3 & 1083 \\
\hline
\end{tabular}

a Regions specific to symbolic ordinal processing of numbers.

${ }^{b}$ Regions specific to nonsymbolic ordinal processing of numbers (see also Figure 3 ).

INS, Insula; L, left; $R$, right.

not as a novel result unto itself, but rather as a means of more precisely defining and visualizing the nature of this anteriorposterior transition as a gradient (the data in Fig. $2 c$ are thus reported in the absence of spatial smoothing to better capture the nature of this gradient).

The top graph in Figure $2 c$ shows average activity for the NumOrd, DotOrd, and control (LumOrd) conditions. All conditions showed a general increase in activity that peaked between processing of symbolic numbers.

Overall, these data are consistent with the notion that assessing ordinality in symbolic and nonsymbolic numbers relies on qualitatively different processes. Overlap was observed only in a prefrontal area not canonically associated with basic number representation. Further analysis indicated this overlap may in fact be an artifact of two separate networks that happen to be anatomically adjacent in that section of cortex. Whole-brain and ROI analyses showed a left-lateralized premotor network specific to symbolic ordinal processing of numbers. By contrast, we observed a large, right-lateralized network for nonsymbolic procanonical number representation areas.

\section{Nonsymbolic number processing}

In previous two sections, we assessed format-general (symbolic and nonsymbolic) neural processing of numbers in the context of cardinal and ordinal judgments (Fig. $1 a$, vertical columns). In this and the subsequent section, we turn our attention to neural regions common to cardinal and ordinal processing in each numerical format separately (Fig. 1a, horizontal rows). For nonsymbolic numbers, the distance-effects in Table 2 were consistent across all cardinal and ordinal conditions, which suggests that cardinal and ordinal assessment in nonsymbolic numbers may be underlain by the same fundamental process. If this is true, then there should be large overlap in canonical number processing cessing of numbers that included both cognitive control and 
Table 5. Anatomical region details for regions specific to symbolic and nonsymbolic ordinal processing of numbers

\begin{tabular}{|c|c|c|c|c|c|c|c|c|c|}
\hline \multirow[b]{2}{*}{ Region } & \multirow[b]{2}{*}{ Condition } & \multicolumn{4}{|c|}{ \% Signal change: mean (SE) } & \multirow[b]{2}{*}{ NumOrd $\neq$ DotOrd } & \multirow[b]{2}{*}{ NumOrd $\neq$ Control } & \multirow[b]{2}{*}{ DotOrd $\neq=$ Control } & \multirow[b]{2}{*}{$2 \times 2 \operatorname{lnt}}$. \\
\hline & & Num0rd & Dot0rd & NumCard & DotCard & & & & \\
\hline \multirow[t]{2}{*}{ PreSMA $^{a}$} & Number & $0.83(0.05)$ & $0.87(0.05)$ & $0.56(0.05)$ & $0.70(0.05)$ & \multirow[t]{2}{*}{$p=0.328$} & \multirow[t]{2}{*}{-- } & \multirow[t]{2}{*}{$p<0.001$} & \multirow[t]{2}{*}{$p=0.598$} \\
\hline & Control & $0.67(0.05)$ & $0.68(0.06)$ & $0.50(0.06)$ & $0.53(0.06)$ & & & & \\
\hline \multirow[t]{2}{*}{ L. PMd ${ }^{a}$} & Number & $0.52(0.07)$ & $0.41(0.08)$ & $0.30(0.07)$ & $0.37(0.08)$ & \multirow[t]{2}{*}{$p=0.006$} & \multirow[t]{2}{*}{-} & \multirow[t]{2}{*}{$p=0.159$} & \multirow[t]{2}{*}{$p=0.006$} \\
\hline & Control & $0.27(0.08)$ & $0.35(0.07)$ & $0.26(0.07)$ & $0.27(0.08)$ & & & & \\
\hline \multirow[t]{2}{*}{ L. PMv ${ }^{a}$} & Number & $0.26(0.08)$ & $0.06(0.07)$ & $0.10(0.07)$ & $0.06(0.07)$ & \multirow[t]{2}{*}{$p<0.001$} & \multirow[t]{2}{*}{-} & \multirow[t]{2}{*}{$p=0.263$} & \multirow[t]{2}{*}{$p<0.001$} \\
\hline & Control & $0.03(0.07)$ & $0.12(0.06)$ & $0.05(0.07)$ & $0.03(0.08)$ & & & & \\
\hline \multirow[t]{2}{*}{ R. IPSa $a^{b}$} & Number & $0.56(0.05)$ & $0.76(0.06)$ & $0.47(0.04)$ & $0.60(0.06)$ & \multirow[t]{2}{*}{$p<0.001$} & \multirow[t]{2}{*}{$p=0.895$} & \multirow[t]{2}{*}{-- } & \multirow[t]{2}{*}{$p<0.001$} \\
\hline & Control & $0.55(0.05)$ & $0.54(0.05)$ & $0.40(0.06)$ & $0.42(0.05)$ & & & & \\
\hline \multirow[t]{2}{*}{$\mathrm{ACCd}^{b}$} & Number & $0.46(0.05)$ & $0.59(0.04)$ & $0.27(0.04)$ & $0.42(0.05)$ & \multirow[t]{2}{*}{$p<0.001$} & \multirow[t]{2}{*}{$p=0.049$} & \multirow[t]{2}{*}{-- } & \multirow[t]{2}{*}{$p=0.010$} \\
\hline & Control & $0.37(0.05)$ & $0.35(0.05)$ & $0.24(0.06)$ & $0.24(0.05)$ & & & & \\
\hline \multirow[t]{2}{*}{ R. DLPFC ${ }^{b}$} & Number & $0.46(0.04)$ & $0.64(0.05)$ & $0.32(0.04)$ & $0.46(0.05)$ & $p<0.001$ & $p=0.708$ & -- & $p<0.001$ \\
\hline & Control & $0.45(0.05)$ & $0.43(0.04)$ & $0.31(0.05)$ & $0.32(0.05)$ & & & & \\
\hline R. IFGa ${ }^{b}$ & Number & $-0.02(0.05)$ & $0.13(0.05)$ & $0.00(0.04)$ & $0.02(0.04)$ & $p=0.002$ & $p=0.490$ & - & $p=0.021$ \\
\hline & Control & $-0.06(0.05)$ & $-0.08(0.05)$ & $0.01(0.05)$ & $-0.07(0.05)$ & & & & \\
\hline L. IFGa ${ }^{b}$ & Number & $-0.09(0.06)$ & $0.02(0.08)$ & $-0.18(0.06)$ & $-0.10(0.06)$ & $p=0.050$ & $p=0.231$ & - & $p=0.065$ \\
\hline & Control & $-0.17(0.06)$ & $-0.22(0.06)$ & $-0.11(0.04)$ & $-0.07(0.06)$ & & & & \\
\hline R. INSa ${ }^{b}$ & Number & $0.39(0.05)$ & $0.53(0.04)$ & $0.23(0.04)$ & $0.37(0.05)$ & $p=0.001$ & $p=0.086$ & - & $p=0.046$ \\
\hline & Control & $0.30(0.05)$ & $0.30(0.05)$ & $0.17(0.06)$ & $0.15(0.05)$ & & & & \\
\hline L. INS $a^{b}$ & Number & $0.34(0.05)$ & $0.45(0.04)$ & $0.18(0.04)$ & $0.31(0.05)$ & $p=0.003$ & $p=0.353$ & -- & $p=0.004$ \\
\hline & Control & $0.30(0.05)$ & $0.22(0.05)$ & $0.12(0.05)$ & $0.15(0.05)$ & & & & \\
\hline
\end{tabular}

Left, Anatomical region details for regions specific to symbolic and nonsymbolic ordinal processing of numbers (see also Figure 3). Right, $p$ values for ROI-level contrasts (see Results, Ordinal processing). Values denoted as are whole-brain contrasts (thus $p<0.005)$. The rightmost column is the $p$ value associated with the 2 (Condition: number, control) $\times 2$ (Format: symbolic, nonsymbolic) interaction. All tests are within-subjects $\left(t_{(32)}, F_{(1,32)}\right)$. $L$, left; R, right.

${ }^{a}$ Regions specific to symbolic ordinal processing of numbers.

${ }^{b}$ Regions specific to nonsymbolic ordinal processing of numbers.

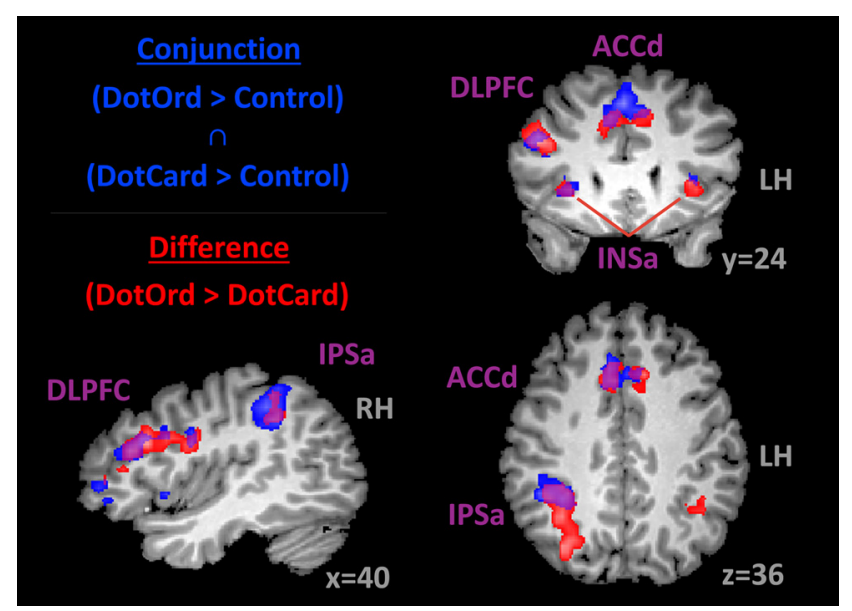

Figure 4. Regions in blue are common to ordinal and cardinal processing of nonsymbolic numbers and are derived from the conjunction of contrasts: (DotOrd $>$ Control) $\cap$ (DotCard $>$ Control). Regions in red showed greater activity for ordinal relative to cardinal processing of nonsymbolic numbers (DotOrd $>$ DotCard). Voxels in purple are the overlap of the conjunction and difference contrasts from above.

areas (IPS) for ordinal and cardinal processing in nonsymbolic numbers. We tested this via the conjunction of the two contrasts: DotOrd $>$ Control $\left(\right.$ LumOrd $_{\text {dot }}$ ) and DotCard $>$ Control (Lum$\left.\operatorname{Card}_{\text {dot }}\right)$. This identified regions that were specific to numerical processing, and common to ordinal and cardinal processing of nonsymbolic numbers (Fig. $1 a$, bottom row). Results are shown in blue in Figure 4; regions details and condition means are given in Table 6. This revealed a primarily right-lateralized set of regions, including the right IPSa, consistent with the notion that both ordinality and cardinality of nonsymbolic numbers involves activation of an area routinely seen for nonsymbolic number processing in general.

How might a single process underlie both ordinal and cardinal assessment (for nonsymbolic numbers)? It may be that ordinality is assessed in dots by iteratively comparing constituent pairs of dot arrays (e.g., "Is the left greater than the middle array; is the middle greater than the right array; is the left greater than the right array?"). That is, in the DotOrd task, one makes multiple cardinal judgments, but in the DotCard task, one makes only a single cardinality judgment. In regions where this is true, one would expect to find greater activity for the DotOrd relative to the DotCard task. The rightmost column in Table 6 shows the results of the contrast (DotOrd > DotCard) in each of the ROIs identified in the conjunction analysis. Notably, right IPSa was one of the regions in which DotOrd activity was significantly greater than DotCard activity $(p<0.001)$. This result was also confirmed at the whole-brain level, which revealed the red regions shown in Figure 4. Voxels colored purple show the extensive spatial overlap for both the conjunction (blue) and contrast (red) analyses, which further underscores the interpretation that nonsymbolic ordinal processing operates via iterative cardinal assessment. Note that this interpretation is also consistent with the anterior-posterior distinction shown in Figure 2: The DotOrd task activates relatively anterior ACC, which is consistent with the need to maintain ongoing cognitive control over multiple, iterative judgments.

\section{Symbolic number processing}

Does ordinal assessment in symbolic numbers also operate via multiple, iterative (symbolic) cardinal judgments? Reversed distance-effects in the NumOrd task and the lack of canonical, parietal areas shown for this task (Fig. 3) argue against this view. Rather, these behavioral effects suggest that cardinal and ordinal assessment in symbolic numbers operate via qualitatively distinct processes. To further test this idea, we examined the degree of overlap for the NumCard and NumOrd conditions at the wholebrain level.

As with nonsymbolic numbers, we tested for overlap of ordinal and cardinal processing in symbolic numbers via the conjunction of the two contrasts: NumOrd $>$ Control $\left(\right.$ LumOrd $\left._{\text {num }}\right)$ and Num- 
Table 6. Region details for regions common to ordinal and cardinal processing of nonsymbolic numbers

\begin{tabular}{|c|c|c|c|c|c|c|c|c|c|c|}
\hline \multirow[b]{2}{*}{ Region } & \multicolumn{3}{|c|}{ Talairach coordinates } & \multirow[b]{2}{*}{ Vol. $\left(\mathrm{mm}^{3}\right)$} & \multirow[b]{2}{*}{ Condition } & \multicolumn{4}{|c|}{ \% Signal change: mean (SE) } & \multirow[b]{2}{*}{ DotOrd $\neq$ DotCard } \\
\hline & $x$ & $y$ & $z$ & & & Num0rd & DotOrd & NumCard & DotCard & \\
\hline \multirow[t]{2}{*}{ R. IPSa } & \multirow[t]{2}{*}{40} & \multirow{2}{*}{-37} & \multirow[t]{2}{*}{41} & \multirow[t]{2}{*}{3702} & Number & $0.53(0.05)$ & $0.73(0.06)$ & $0.45(0.04)$ & $0.59(0.06)$ & \multirow[t]{2}{*}{$p<0.001$} \\
\hline & & & & & Control & $0.53(0.05)$ & $0.52(0.05)$ & $0.38(0.06)$ & $0.39(0.05)$ & \\
\hline \multirow[t]{2}{*}{ R. PPC } & \multirow[t]{2}{*}{27} & \multirow[t]{2}{*}{-72} & \multirow[t]{2}{*}{26} & \multirow[t]{2}{*}{473} & Number & $0.49(0.08)$ & $0.79(0.09)$ & $0.40(0.08)$ & $0.71(0.08)$ & \multirow{2}{*}{$p=0.149$} \\
\hline & & & & & Control & $0.58(0.08)$ & $0.61(0.08)$ & $0.37(0.08)$ & $0.48(0.09)$ & \\
\hline \multirow[t]{2}{*}{$\mathrm{ACCd}$} & \multirow[t]{2}{*}{2} & \multirow[t]{2}{*}{19} & \multirow[t]{2}{*}{42} & \multirow[t]{2}{*}{4707} & Number & $0.50(0.05)$ & $0.63(0.05)$ & $0.30(0.04)$ & $0.46(0.05)$ & \multirow[t]{2}{*}{$p<0.001$} \\
\hline & & & & & Control & $0.40(0.06)$ & $0.39(0.05)$ & $0.26(0.06)$ & $0.25(0.06)$ & \\
\hline \multirow[t]{2}{*}{ R. DLPFCa } & \multirow[t]{2}{*}{39} & \multirow[t]{2}{*}{30} & \multirow[t]{2}{*}{22} & \multirow[t]{2}{*}{2166} & Number & $0.37(0.05)$ & $0.56(0.05)$ & $0.26(0.04)$ & $0.40(0.05)$ & \multirow[t]{2}{*}{$p<0.001$} \\
\hline & & & & & Control & $0.38(0.05)$ & $0.36(0.05)$ & $0.24(0.06)$ & $0.23(0.05)$ & \\
\hline \multirow[t]{2}{*}{ R. DLPFCp } & \multirow[t]{2}{*}{45} & \multirow[t]{2}{*}{4} & 27 & 1192 & Number & $0.73(0.06)$ & $0.92(0.07)$ & $0.53(0.05)$ & $0.75(0.07)$ & $p<0.001$ \\
\hline & & & & & Control & $0.73(0.07)$ & $0.72(0.07)$ & $0.56(0.07)$ & $0.55(0.07)$ & \\
\hline R.IFGa & 39 & 44 & 2 & 343 & Number & $0.00(0.06)$ & $0.18(0.06)$ & $0.05(0.04)$ & $0.09(0.06)$ & $p=0.051$ \\
\hline & & & & & Control & $0.02(0.05)$ & $-0.04(0.06)$ & $0.05(0.06)$ & $-0.06(0.05)$ & \\
\hline R. INSa & 31 & 18 & 4 & 1667 & Number & $0.38(0.05)$ & $0.50(0.04)$ & $0.24(0.04)$ & $0.38(0.05)$ & $p<0.001$ \\
\hline & & & & & Control & $0.31(0.05)$ & $0.30(0.05)$ & $0.18(0.06)$ & $0.15(0.04)$ & \\
\hline L. INSa & -32 & 18 & 4 & 721 & Number & $0.41(0.05)$ & $0.52(0.04)$ & $0.24(0.04)$ & $0.39(0.05)$ & $p=0.001$ \\
\hline & & & & & Control & $0.35(0.05)$ & $0.30(0.05)$ & $0.19(0.06)$ & $0.19(0.05)$ & \\
\hline L. FFGp & -43 & -68 & -5 & 397 & Number & $0.83(0.09)$ & $0.84(0.08)$ & $0.77(0.08)$ & $0.94(0.09)$ & $p=0.042$ \\
\hline & & & & & Control & $0.82(0.09)$ & $0.74(0.09)$ & $0.68(0.08)$ & $0.67(0.08)$ & \\
\hline L.CLS & -9 & -74 & 8 & 624 & Number & $-0.23(0.08)$ & $-0.14(0.08)$ & $-0.21(0.06)$ & $-0.08(0.08)$ & $p=0.137$ \\
\hline & & & & & Control & $-0.22(0.08)$ & $-0.27(0.08)$ & $-0.33(0.08)$ & $-0.32(0.07)$ & \\
\hline
\end{tabular}

Region details for regions common to ordinal and cardinal processing of nonsymbolic numbers (see also blue regions in Figure 4). Anatomical information is shown on the left and mean neural activity on the middle. Activity is shown for each experimental (Number) and control condition. The rightmost column shows the result of testing whether DotOrd activity was greater than DotCard activity in a given region (see also red and purple regions in Figure 4). PPC, Posterior parietal cortex; FFG, fusiform gyrus; L, left; $R$, right.

Card $>$ Control $\left(\right.$ LumCard $\left._{\text {num }}\right)$. In theory, this should identify regions specific to numerical processing, and common to ordinal and cardinal processing of symbolic numbers (Fig. $1 a$, top row). There were no significant regions at the threshold used for our other whole-brain contrasts $(p<0.005, \alpha<.01)$. While this is consistent with the hypothesis that ordinality and cardinality operate differently for numerals, it is nevertheless an argument from a null effect. On the one hand, it is unlikely that lack of power is the main issue as we found many highly significant regions for nonsymbolic numbers in the previous section. On the other hand, there may be regions in the current analysis evident only on the slightly less significant side of the arbitrary threshold adopted here.

To address this, we lowered the threshold to $p<0.05$, uncorrected, and assessed the percentage of active voxels seen for the $\mathrm{Nu}$ mOrd $>$ Control $\left(\right.$ LumOrd $\left._{\text {num }}\right)$ contrast that was also active at that threshold for the NumCard $>$ Control $\left(\right.$ LumCard $_{\text {num }}$ ) contrast. Even at this far more liberal threshold, only 1.1\% of NumOrd voxels were also active for the NumCard task, which is similar to what one would predict by chance $(2.5 \%)$ (Šidak, 1967). (Using these same criteria for dots, $40.2 \%$ of DotOrd voxels were also active for the DotCard task.) In sum, we find no evidence that assessing ordinality and cardinality in symbolic numbers rely on similar brain regions.

\section{Discussion}

By focusing on ordinality (an oft overlooked property of number), we found clear behavioral and neural evidence distinguishing symbolic and nonsymbolic representations of number. Analyses of both behavioral and neural data demonstrated that symbolic numberordering is the "odd man out," when compared with symbolic cardinal processing and nonsymbolic number processing in general. Behavioral distance-effects were reversed only when assessing ordinality in symbolic numbers; canonical distance effects were observed for all other conditions. Further, symbolic number-ordering was the only numerical task that did not show number-specific activity (i.e., greater than control) in right IPSa- a region associated with basic number representation (Tables $3 a, 5 b, 6$ ). Instead, activity in left premotor cortex was specifically associated with symbolic numberordering, suggesting complex visuomotor associations perhaps re- lated to the count-list play a role in symbolic but not nonsymbolic ordinal processing. These associations may be unavailable to nonsymbolic number-ordering. Instead, for nonsymbolic number, recruitment of cognitive control areas along with a high degree of overlap with nonsymbolic cardinal processing indicate that ordinality is assessed via iterative cardinality judgments. This contrasts with a striking lack of neural overlap between ordinal and cardinal judgments in symbolic numbers. Taken together with the opposing distance-effects for ordinal and cardinal processing of symbolic number, this suggests that symbolic number processing varies substantially with computational context.

This latter point is consistent with the notion that symbolic number representation depends on the nature of the computational demands (Cipolotti et al., 1995; Delazer and Butterworth, 1997; Turconi and Seron, 2002). Intuitively, the number 6 is in one sense equally $5+1,7-1, \frac{18}{3}$, and $\sqrt[3]{216}$; but the functional import of each of these representations is driven strongly by context. At the broadest level, the meaning of 6 may thus be determined by both its relation to the other symbolic numbers and the computational context in which it rests. This is in keeping with the view that the meaning of symbolic numbers is fundamentally tied to their relations with other symbolic numbers (Wiese, 2003; Nieder, 2009). At the very least, our results indicate that ordinality and cardinality are qualitatively different processes in symbolic numbers. In other words, it is not just that the processing of ordinality depends on representational format (symbolic vs nonsymbolic), even within symbolic numbers, ordinal processing appears to be distinct from cardinal processing.

By contrast, such representational flexibility appears to be unavailable to nonsymbolic numbers. To be clear, we are not claiming that ordinal assessment in nonsymbolic numbers is impossible. Indeed, performance on the DotOrd task was well beyond chance (mean error-rate: $18.2 \%$, standard error $=1.1 \%$; chance $=50 \%$ ). Rather, our claim is that the manner in which this occurs is fundamentally different from that in symbolic numbers. Ordinal assessment in nonsymbolic numbers appears to operate by iteratively reusing the same neural processes that underlie cardinal assessment. 
Similarly, as canonical distance-effects in the Ord-Mix condition indicate, it would be unwise to conclude that ordinal assessment in symbolic numbers cannot operate in this manner. Rather, both the neural results and the reversed distance-effects in the Ord-Ord condition seen for symbolic but not nonsymbolic numbers converge on the conclusion that ordinal processing of symbolic numbers presents a mechanism unavailable to nonsymbolic numbers.

One possibility is that this mechanism involves retrieval from the highly rehearsed count-list. Consistent with this view, whole-brain and ROI analyses indicated ordinal processing of symbolic numbers was specific to two left-lateralized premotor regions: PMd and PMv (Fig. 3). More detailed analysis of the ACCdp regions showing overlap for symbolic and nonsymbolic number-ordering indicated that this reflects the posterior edge of a more anterior ACC region for nonsymbolic ordering, and the anterior edge of a more posterior midline region (moving into premotor cortex) for symbolic ordering. This posterior shift might indicate that ordinal assessment in symbolic numbers-but not nonsymbolic numbers-relies increasingly on proceduralized retrieval processes. SMA has been shown to be important for sequential order processing (Gerloff et al., 1997; Tanji, 2001), and PMd and PMv are involved in retrieval of visuospatial action-plans in response to overlearned symbolic associations (Grafton et al., 1998; Wise and Murray, 2000; Hoshi and Tanji, 2007; O'Shea et al., 2007), as one would expect when retrieving from a highly proceduralized count-list. Without such retrieval processes available to nonsymbolic ordinal assessment, this process might rely on more anterior ACC tissue, which is more often seen in regulating complex ongoing cognitive demands (Botvinick et al., 2004; Sheth et al., 2012). One interesting prediction from this interpretation is that one should see more efficient ordinal assessment for any overlearned, regularly rehearsed numerical sequence (e.g., one's phone number or postal code, multiples of 7 for American football fans), but only when these quantities are presented symbolically.

As seen in Table 2 and Figure $2 a$, for cardinality judgments, we replicated prior results in showing both similar behavioral responses (qualitatively similar distance-effects) and neural overlap between symbolic and nonsymbolic numbers in a canonical numberprocessing area (Dehaene et al., 2008; Nieder and Dehaene, 2009) (right IPSa). However, this was limited to cardinal processing of numbers (NumCard and DotCard tasks). Thus, we are not arguing that there is no overlap whatsoever between symbolic and nonsymbolic number processing. Rather, our data indicate that such overlap is largely limited to cardinal processing of numbers.

Our results revealed a high degree of overlap for nonsymbolic ordinal and cardinal processing (especially in the IPS), and are thus consistent with previous work showing that representations of nonsymbolic numbers are relatively invariant over different computational contexts (Stanescu-Cosson et al., 2000; Venkatraman et al., 2005, 2006; such as number comparison and approximate arithmetic). Given this representational inflexibility, ordinal assessment of nonsymbolic numbers thus appears to be restricted to operating via iterative cardinality assessments (i.e., comparing each pair of numbers in succession). On a broader note, we view the case of symbolic numbers to be one example among many demonstrating the fundamental trade-off between symbolic and nonsymbolic representation. Nonsymbolic representations may be better grounded in intuitive, analog, perceptual processes (Kontra et al., 2012), but this may come at the cost of associative inflexibility - that is, the ability to rapidly change meaning depending on context (Crutch and Warrington, 2010). By contrast, our results indicate a high degree of representational flexibility is accorded to symbolic numbers, although this may come at the cost of being estranged from a more intuitive sense of quantity or magnitude available to nonsymbolic numbers (Lyons et al., 2012).

On the surface, our results appear to contrast with two previous studies that concluded left IPS is important for symbolic ordinal processing. First, Fias et al. (2007) showed that two-digit numeral comparison and letter comparison (alphabetical order) tasks coactivated (each relative to a luminance-dimming detection task) FFGp, SMA, IPSa, IPSp, PMd, and PMv, with slightly stronger effects for the latter four regions in the left-hemisphere. The authors concluded that canonical number-processing areas (IPS) generalize to nonnumerical ordinal processing (of symbolic stimuli) as well. It is interesting that several of these other areas overlap with the premotor regions we found to be specific to the NumOrd task in the current dataset, which may suggest that these regions process ordinal relations in symbols more generally. On the other hand, it is worth noting that Fias et al. (2007) used comparison tasks, and we have already noted how our results strongly suggest that symbolic number processing is quite sensitive to computational context. Hence, an approach where subjects explicitly judge the order of three items (e.g., letters, numbers, etc.) would be a stronger test of ordinal processing than comparing just two items (Franklin and Jonides, 2009; Lyons and Beilock, 2009, 2011).

Second, Franklin and Jonides (2009) presented ordinal stimuli in a manner similar to the current study, with triplets in increasing, decreasing, or mixed (left-right) order. The authors showed that processing of symbolic numerical distance in the left IPS is sensitive to computational context. Namely, left IPS showed greater activity for close relative to far-distance trials in a two-digit comparison task ("Which of 2 two-digit numbers represents the greater quantity?") and greater activity for the reverse contrast (far relative to closedistance trials) in the three-digit ordering task. Our results also demonstrate that symbolic numbers are treated differently as a function of computational context. On the other hand, Franklin and Jonides (2009) assessed activity during ordinal processing of symbolic numbers only as a function of distance-effects (e.g., far $>$ close and close $>$ far). As the reversible nature of distance-effects in both their study and ours makes clear, the relation between distance-effects and representation in symbolic numbers is not straightforward [for further evidence and discussion to this end, see the studies by Verguts and van Opstal (2005), van Opstal et al. (2008). It is thus difficult to infer the exact meaning of the left IPS activity Franklin and Jonides (2009) showed in terms of symbolic number representation. In the current study, we included control tasks to isolate both numberspecific and task-specific (i.e., ordinal versus cardinal judgments) neural processing. In doing so, even using a block design and large sample designed to maximize statistical power, we did not find any section of the parietal lobe to be centrally involved in processing ordinality in symbolic numbers (note that this was true even at the quite liberal threshold of $p<0.05$ ). On the other hand, our results showed that a network of premotor regions in the left hemisphere are the most likely to be central to processing ordinality in symbolic numbers.

In conclusion, we show that considering ordinality points to striking differences between symbolic and nonsymbolic numbers. The previously reported connection between symbolic and nonsymbolic representations of number appears to be restricted to cardinal processing. A deeper understanding of ordinal processing in symbolic numbers may shed further light on the flexibility and precision of representation available to symbolic but not nonsymbolic numbers. In sum, considering ordinality-a relatively overlooked property of numbers - may prove crucial for understanding the power of representing numbers symbolically. 


\section{References}

Botvinick MM, Cohen JD, Carter CS (2004) Conflict monitoring and anterior cingulate cortex: an update. Trends Cogn Sci 8:539-546. CrossRef Medline

Buckley PB, Gillman CB (1974) Comparisons of digits and dot patterns. J Exp Psychol 103:1131-1136. CrossRef Medline

Cipolotti L, Warrington EK, Butterworth B (1995) Selective impairment in manipulating Arabic numerals. Cortex 31:73-86. CrossRef Medline

Crutch SJ, Warrington EK (2010) The differential dependence of abstract and concrete words upon associative and similarity-based information: complementary semantic interference and facilitation effects. Cogn Neuropsychol 27:46-71. CrossRef Medline

Dehaene S (2008) Symbols and quantities in parietal cortex: elements of a mathematical theory of number representation and manipulation. In: Sensorimotor foundations of higher cognition (attention and performance) (Haggard, P and Rossetti, Y, Eds), pp 527-574. New York: Oxford UP.

Dehaene S, Piazza M, Pinel P, Cohen L (2003) Three parietal circuits for number processing. Cogn Neuropsychol 20:487-506. CrossRef Medline

Delaney HD, Maxwell SE (1981) On using analysis of covariance in repeated measures designs. Multivar Behav Res 16:105-123. CrossRef

Delazer M, Butterworth B (1997) A dissociation of number meanings. Cogn Neuropsychol 14:613-636. CrossRef

Diester I, Nieder A (2007) Semantic associations between signs and numerical categories in the prefrontal cortex. PLoS: Biol 5:e294. CrossRef Medline

Diester I, Nieder A (2010) Numerical values leave a semantic imprint on associated signs in monkeys. J Cogn Neurosci 22:174-183. CrossRef Medline

Eger E, Michel V, Thirion B, Amadon A, Dehaene S, Kleinschmidt A (2009) Deciphering cortical number coding from human brain activity patterns. Curr Biol 19:1608-1615. CrossRef Medline

Fias W, Lammertyn J, Reynvoet B, Dupont P, Orban GA (2003) Parietal representation of symbolic and nonsymbolic magnitude. J Cogn Neurosci 15:47-56. CrossRef Medline

Fias W, Lammertyn J, Caessens B, Orban GA (2007) Processing of abstract ordinal knowledge in the horizontal segment of the intraparietal sulcus. J Neurosci 27:8952-8956. CrossRef Medline

Forman SD, Cohen JD, Fitzgerald M, Eddy WF, Mintun MA, Noll DC (1995) Improved assessment of significant activation in functional magnetic resonance imaging (fMRI): use of a cluster-size threshold. Magn Reson Med 33:636-647. CrossRef Medline

Franklin MS, Jonides J (2009) Order and magnitude share a common representation in parietal cortex. J Cogn Neurosci 21:2114-2120. CrossRef Medline

Friston KJ, Holmes AP, Worsley KJ, Poline JP, Frith CD, Frackowiak RSJ (1994) Statistical parametric maps in functional imaging: a general linear approach. Hum Brain Mapp 2:189-210. CrossRef

Gerloff C, Corwell B, Chen R, Hallett M, Cohen LG (1997) Stimulation over the human supplementary motor area interferes with the organization of future elements in complex motor sequences. Brain 120:1587-1602. CrossRef Medline

Gilmore CK, McCarthy SE, Spelke ES (2010) Non-symbolic arithmetic abilities and mathematics achievement in the first year of formal schooling. Cognition 115:394-406. CrossRef Medline

Grafton ST, Fagg AH, Arbib MA (1998) Dorsal premotor cortex and conditional movement selection: a PET functional mapping study. J Neurophysiol 79:1092-1097. Medline

Halberda J, Mazzocco MM, Feigenson L (2008) Individual differences in non-verbal number acuity correlate with maths achievement. Nature 455: 665-668. CrossRef Medline

Hoshi E, Tanji J (2007) Distinctions between dorsal and ventral premotor areas: anatomical connectivity and functional properties. Curr Opin Neurobiol 17:234-242. CrossRef Medline

Kontra C, Goldin-Meadow S, Beilock SL (2012) Embodied learning across the life span. Topics Cogn Sci 4:731-739. CrossRef Medline

Kriegeskorte N, Simmons WK, Bellgowan PS, Baker CI (2009) Circular analysis in systems neuroscience: the dangers of double dipping. Nat Neurosci 12:535-540. CrossRef Medline

Lyons IM, Beilock SL (2009) Beyond quantity: individual differences in working-memory and the ordinal understanding of numerical symbols. Cognition 113:189-204. CrossRef Medline

Lyons IM, Beilock SL (2011) Numerical ordering ability mediates the relation between number-sense and arithmetic competence. Cognition 121: 256-261. CrossRef Medline

Lyons IM, Ansari D, Beilock SL (2012) Symbolic estrangement: evidence against a strong association between numerical symbols and the quantities they represent. J Exp Psychol Gen 141:635-641. Medline

McCrink K, Spelke ES (2010) Core multiplication in childhood. Cognition 116:204-216. CrossRef Medline

Moyer RS, Landauer TK (1967) Time required for judgments of numerical inequality. Nature 215:1519-1520. CrossRef Medline

Nieder A (2009) Prefrontal cortex and the evolution of symbolic reference. Curr Opin Neurobiol 19:99-108. CrossRef Medline

Nieder A, Dehaene S (2009) Representation of number in the brain. Annu Rev Neurosci 32:185-208. CrossRef Medline

Oldfield RC (1971) The assessment and analysis of handedness: the Edinburgh inventory. Neuropsychologia 9:97-113. CrossRef Medline

O'Shea J, Johansen-Berg H, Trief D, Göbel S, Rushworth MF (2007) Functionally specific reorganization in human premotor cortex. Neuron 54: 479-490. CrossRef Medline

Piazza M, Pinel P, Le Bihan D, Dehaene S (2007) A magnitude code common to numerosities and number symbols in human intraparietal cortex. Neuron 53:293-305. CrossRef Medline

Piazza M, Facoetti A, Trussardi AN, Berteletti I, Conte S, Lucangeli D, Dehaene S, Zorzi M (2010) Developmental trajectory of number acuity reveals a severe impairment in developmental dyscalculia. Cognition 116 : 33-41. CrossRef Medline

Sheth SA, Mian MK, Patel SR, Asaad WF, Williams ZM, Dougherty DD, Bush G, Eskandar EN (2012) Human dorsal anterior cingulate cortex neurons mediate ongoing behavioural adaptation. Nature 488:218-221. CrossRef Medline

Šidak Z (1967) Rectangular confidence regions for the means of multivariate normal distributions. J Am Stat Assoc 62:626-633. CrossRef

Stanescu-Cosson R, Pinel P, van De Moortele PF, Le Bihan D, Cohen L, Dehaene S (2000) Understanding dissociations in dyscalculia: a brain imaging study of the impact of number size on the cerebral networks for exact and approximate calculation. Brain 123:2240-2255. CrossRef Medline

Tanji J (2001) Sequential organization of multiple movements: involvement of cortical motor areas. Ann Rev Neurosci 24:631-651. CrossRef Medline

Turconi E, Seron X (2002) Dissociation between order and quantity meanings in a patient with Gerstmann Syndrome. Cortex 38:911-914. CrossRef

Turconi E, Campbell JI, Seron X (2006) Numerical order and quantity processing in number comparison. Cognition 98:273-285. CrossRef Medline

Unsworth N, Heitz RP, Schrock JC, Engle RW (2005) An automated version of the operation span task. Behavior Research Methods 37:498-505. CrossRef Medline

van Opstal F, Gevers W, De Moor W, Verguts T (2008) Dissecting the symbolic distance effect: comparison and priming effects in numerical and nonnumerical orders. Psychon Bull Rev 15:419-425. CrossRef Medline

Venkatraman V, Ansari D, Chee MW (2005) Neural correlates of symbolic and non-symbolic arithmetic. Neuropsychologia 43:744-753. CrossRef Medline

Venkatraman V, Siong SC, Chee MW, Ansari D (2006) Effect of language switching on arithmetic: a bilingual FMRI study. J Cogn Neurosci 18:6474. CrossRef Medline

Verguts T, Fias W (2004) Representation of number in animals and humans: a neural model. J Cogn Neurosci 16:1493-1504. CrossRef Medline

Verguts T, van Opstal F (2005) Dissociation of the distance effect and size effect in one-digit numbers. Psychon Bull Rev 12:925-930. CrossRef Medline

Wagner JB, Johnson SC (2011) An association between understanding cardinality and analog magnitude representations in preschoolers. Cognition 119:10-22. CrossRef Medline

Wiese H (2003) Iconic and non-iconic stages in number development: the role of language. Trends Cogn Sci 7:385-390. CrossRef Medline

Wise SP, Murray EA (2000) Arbitrary associations between antecedents and actions. Trends Neurosci 23:271-276. CrossRef Medline 